\title{
Odour and Proustian Memory: Reduction of Context- dependent Forgetting and Multiple Forms of Memory
}

\author{
AMANDA PARKER, $*$ HENNY NGU \\ and HELEN J. CASSADAY
}

University of Nottingham, $U K$

\begin{abstract}
SUMMARY
In Experiment 1, the reintroduction of the same ambient odour (lemon or lavender) improved performance four weeks later in both free recall and recognition of a word list. This was a cross-over design that allowed direct comparison between congruent and incongruent odour conditions. A further comparison with an additional group showed that memory was not improved by the presence of a different odour. Experiment 2 investigated the effect of two odour cues (lemon and lavender) in the same cross-over design using three learning and memory tests: (1) free recall of a word list; (2) problem solving; and (3) spatial learning. While recall of the word list and spatial learning were best when the same odour was present at both learning and test, there was no such context-dependent effect for the problem-solving task. However, the presence of the lavender odour at test improved performance in the problem-solving task, irrespective of the odour present at the first exposure. Thus although lavender had some effect on problem solving, we saw context-dependent retrieval only in free recall and spatial learning. We discuss the implications of this dissociation. Copyright (C) 2001 John Wiley \& Sons, Ltd.
\end{abstract}

\section{GENERAL INTRODUCTION}

The notion that memory is improved by the provision of reminders is a familiar one and, for many people, smell is particularly evocative. In the famous episode of 'la madeleine', Marcel Proust, in Remembrance of Things Past, shows how when memories have faded, smell and taste can be potent cues for memories of associated experiences. This effect (also evoked by music) has become known as Proustian memory. Environmental context (EC) effects have also been studied experimentally using exposure to different environmental stimuli in a cross-over design that allows controlled comparison between congruent and incongruent EC. For example, a study of divers found that information learned on land was better recalled on land rather than under water, whereas information learned under water was better remembered under water (Godden and Baddeley, 1975). Odour has similarly been shown to benefit retrieval, not simply because of some activating or motivational effect, because the same odour must be present at both learning and retrieval (e.g. Eich, 1978; Schab, 1990; Smith et al., 1992). However, such context-dependent retrieval can be difficult to reproduce in the laboratory and the available experimental results are mixed (see Fernandez and Glenberg, 1985; Smith, 1988). In part, these inconsistencies can be

\footnotetext{
* Correspondence to: Dr A. Parker, School of Psychology, University of Nottingham, University Park,
} Nottingham NG7 2RD, UK. E-mail: aep@psychology.nottingham.ac.uk 
accounted for by variation in the experimental procedures adopted (Eich, 1980; Smith, 1988), and making the rooms in EC experiments as different from each other as possible may help to produce a significant difference between groups (Mayes et al., 1981). Ambient odour and music cues are one way in which this can be achieved (Mayes et al., 1981; Parker and Gellatly, 1997).

The present study is concerned with the effect of odour as an aspect of EC. Previous studies of memory for odours have shown that although people recognize pictures better than odours, the forgetting of odours is considerably slower over time (Engen and Ross, 1973; Lawless and Cain, 1975). Similarly, Goldman and Seamon (1992) reported significant retention for odours not experienced since childhood, such as that of children's toys. The folk wisdom that odours may prompt memory of emotionally laden experiences (Laird, 1935) also receives some experimental support (Rubin et al., 1984). However, despite the fact that odours are so well-remembered, subjects are less accurate in attaching appropriate names or verbal labels to particular smells ('the tip-of-the-nose' effect). Memory for odours can be improved using certain encoding strategies such as generating odour names and associating the odour with a life episode that has been evoked (Lyman and McDaniel, 1986, 1990). That odours can serve as cues for autobiographical memories has recently been shown by Aggleton and Waskett (1999), who utilized the unusual odours presented at a Viking museum in a test of events which had occurred on a previous visit to the museum several years ago.

A better understanding of the circumstances under which context-dependent memory can be demonstrated will shed light on its mechanisms. For example, the balance of findings suggests that significant effects are quite often found with recall, but not usually found with recognition tests (for a review see Smith, 1988). A proposed explanation for this effect is the 'outshining hypothesis', described by Smith (1988); 'Outshining is an application of the idea that a heavenly body which is visible on a moonless night is more difficult to see when there is a full moon, and is completely outshone in the daytime by the sun. Similarly, an initially encoded EC can cue memory when better cue sources are absent (i.e. they were not encoded or they are missing from the test information), but EC cues can be completely outshone when better cues are present. Outshining emphasizes the relativity of EC cueing; a cue's effectiveness depends on the presence of better cues' (Smith, 1988, p. 19). Under normal experimental conditions, recognition tests give subjects high-quality cues which obscure the less powerful effects of EC cues. Eich (1980) presents a similar argument.

The present experiments set out to examine systematically the role of odour as an aspect of EC. We used a variety of learning and memory tasks given the evidence of dissociations between the effects of EC manipulation on various forms of explicit and implicit memory (Parker, 1994; Parker and Gellatly, 1997; Parker et al., 1999; Smith, 1988), and gathered evidence from two non-explicit tasks not tested before using odour context manipulations. Where odour works as a cue for memory retrieval, this will have practical implications for the reduction of context-dependent forgetting by the use of odour cueing (Parker and Gellatly, 1997), including possible medical and therapeutic uses.

\section{EXPERIMENT 1}

This study tested recall and recognition memory for a word list learned incidentally in a naturalistic environment. For recall, it was predicted that the group which 
received the same ambient odour cue at both study and test would recall significantly more words than subjects in the incongruent ('crossed') conditions. We included a test of recognition memory because longer-term EC effects cued by an odour have not been examined previously, and weakening of copy cue strength (Eich, 1980) over time may allow the effects of EC cues to emerge on the basis of the outshining hypothesis (Smith, 1988).

\section{Method}

\section{Subjects}

Ninety first-year psychology undergraduates participated in the experiment. They were randomly assigned to experimental conditions.

\section{Stimuli and apparatus}

The ambient odourant used was either lemon or lavender essential oil (Amphora Aromatics Limited). The amount of oil needed and period of diffusion before a subject was allowed to enter was predetermined by a pilot study, using the judgement of three independent individuals to find a comfortable 'ambient' level. This was provided by $0.2 \mathrm{ml}$ of oil (measured using a $1 \mathrm{ml}$ syringe) and placed on a flat dish. The dish was placed under the table and the odour was allowed to diffuse throughout the room for 15 minutes. On completion of the study or memory task, clearance of the odourant was accomplished with two fans and the opening of several windows.

Each of the two word-lists for the study phase contained 30 nouns, taken from the norms of Graf and Williams (1987). The words were balanced across lists for frequency, using the norms of Kucera and Francis (1967). The recognition sheet contained all 60 words. Recognition scores were calculated as (hits-false alarms).

\section{Design and procedure}

Subjects took part in the first part of the experiment immediately before the first laboratory session of their degree. Four weeks later, in the same room, again at the start of laboratory class, subjects completed a free recall test, then a recognition test. An ambient odourant was either present or not present during the study phase. The test phase was in the same room, and either the same odour as at study, or a different odour, or no odour was present. Subjects were tested in groups of 9, and one group in each condition which received an odourant was exposed to lemon odour, the other to lavender.

During the study phase, participants in all conditions were given one of the two word study lists, given randomly, rating each word for pleasantness and for familiarity, then stating the number of vowels contained in the word. On being given the study list, participants were told to read the instructions at the top of the sheet and fill in the boxes carefully, without sacrificing accuracy for speed. No reference to a later memory test was made. The study task was completed without time restrictions, and no participant took longer than 8 minutes.

Four weeks later, participants were tested in the same laboratory. Depending on subject group, the room either had, or did not have, the same ambient odour that had been present during the study phase. In the additional condition, an alternative ambient odour was presented, providing a further comparison extra to the balanced 
cross-over design. Subjects were asked if they would take part in a test of their memory for the word list they had studied in their first laboratory class in the department. On agreeing, they were all given a recall test for the words on the study list, with the sheet instructions asking them to write down as many words from the study list as possible. It was stated that there would not be a time restriction. Following this subjects were given a recognition test sheet, the instructions on which told them that they should circle all words that were on the study list.

\section{Results}

Free recall

The mean proportion of words recalled is shown in Table 1(A). A $2 \times 2$ ANOVA showed that there was a main effect of odour present at study $(F(1,68)=5.49$, $p<0.025)$, but no effect of odour present at test $(F<1)$. There was also a significant interaction between odour at study and at test, consistent with a facilitative effect of context reinstatement $(F(1,68)=4.96, p<0.05)$.

To see whether the positive effect of odour might simply be due to some activating effect, a $t$-test was used to compare the scores of the group who experienced the reinstated odour with an additional group who received the different odour at test. The group who experienced the same odour at both study and test were significantly better at recalling the word list $(t(17)=1.39, p<0.05$, one-tailed).

\section{Recognition}

The mean proportion of words recognized is shown in Table $1(\mathrm{~B})$. The $2 \times 2$ ANOVA revealed a main effect of odour present at study $(F(1,68)=6.03, p<0.025)$, but no effect of odour present at test $(F<1)$. There was also a significant interaction between odour at study and at test, consistent with a facilitative effect of context reinstatement $(F(1,68)=6.46, p<0.01)$.

A $t$-test comparing the scores of the group who experienced the reinstated odour with an additional group who received the different odour at test showed that the

Table 1.

\begin{tabular}{lcc}
\hline & \multicolumn{2}{c}{ Study condition } \\
\cline { 2 - 3 } Test condition & $\begin{array}{c}\text { Odour } \\
\text { present }\end{array}$ & $\begin{array}{c}\text { No odour } \\
\text { present }\end{array}$ \\
\hline
\end{tabular}

(A) Mean $( \pm \mathrm{SD})$ words recalled in Experiment 1

(all $N \mathrm{~s}=18$ )

Odour present

No odour

Different odour
$5.28( \pm 1.93)$

$4.03( \pm 1.72)$

$4.33( \pm 2.14)$
$3.11( \pm 2.17)$

$4.28( \pm 2.19)$

(B) Mean $( \pm \mathrm{SD})$ words recognized in Experiment 1

(all $N \mathrm{~s}=18)$

Odour present

No odour

$10.28( \pm 2.72)$

$7.06( \pm 2.36)$

Different odour

$8.11( \pm 2.91)$

$8.45( \pm 2.75)$

$8.17( \pm 2.89)$ 
group who experienced the same odour at both study and test were significantly better at recognition of the word list $(t(17)=2.01, p<0.025$, one-tailed $)$.

\section{Discussion}

For tests of both recall and recognition, the interaction between the same odour at study and at test produced a facilitatory effect of the reinstated odour. This demonstration of a context-dependent effect in recognition memory replicates Smith (1986), but as recognition followed recall testing in the same subjects here, further experimentation is still required in this area to establish this effect fully. While recognition memory is typically insensitive to context-dependent effects (Smith et al., 1978; Eich, 1980), these can be demonstrated under certain circumstances, such as when an incidental short-term memory task is used in the study phase (Smith, 1986), or with a long study-test interval, as shown here. This main effect of odour at study in the recognition test is consistent with an activational or motivational effect of odour (cf. Schab, 1990). However, the fact that a different odour cue was not sufficient to induce context-dependent retrieval suggests that non-specific activational effects do not mediate the effects of the reminder. Only the specific odour present at study worked as a reminder, memory was not reinstated by the alternate odour.

\section{EXPERIMENT 2}

Experiment 2 investigated the effects of odour as a contextual cue in three tasks: free recall, procedural learning and spatial memory. One of the earliest accounts of context-dependent learning was provided by the philosopher John Locke (1690), who described a man who learned to dance in a room which contained a large trunk: 'The idea of this remarkable piece of household stuff had so mixed itself with the turns and steps of all his dances, that although in that chamber he could dance excellently well, yet it was only while the trunk was there' (pp. 339-340). However, despite the fact that this early anecdote describes an effect on procedural learning, there have been few, if any, systematic context-dependent studies of procedural learning. Similarly, while it is known that other mammals (e.g. rats; Nigrosh et al., 1995) are particularly sensitive to odour and this most likely contributes to their good spatial abilities, there has been little, if any, investigation of context-dependent effects in human spatial learning. However, a recent study by Chun and Phelps (1999) has found that savings in a visual search task is enhanced by the presence of contextual cues in normal control subjects, suggesting that spatial implicit memory may benefit from contextual cues.

Experiment 2 consisted of two sessions, study and test, separated by a 24-hour interval. The free recall task was a word-list; problem solving was measured using two variants of the Tower of Hanoi task (one concrete, one abstract); the spatial task was a supplementary adult level Porteus maze test (Porteus, 1965). Subjects completed the same maze test in both sessions. Although there is some evidence from the neuropsychological literature that the tasks selected depend on different brain systems (e.g. Mayes, 1988; Squire, 1992), much debate currently exists as to the precise regions involved in explicit and implicit tasks, and whether spatial and non-spatial tasks might involve different brain regions independently of the explicit-implicit distinction. 
Our intention here is to test the generality of any context-dependent effect in normal subjects, under a variety of conditions, which are of particular relevance to applied settings.

\section{Method}

\section{Subjects}

Forty-eight undergraduate subjects participated in this experiment. They were randomly assigned to experimental conditions. In addition, a small pilot study $(n=4)$ was used to check that subjects understood the instructions and that none of the tasks was too easy or difficult, so that ceiling and floor effects were avoided.

\section{Stimuli and apparatus}

The experiment was carried out in an experimental cubicle ( $70 \mathrm{ft}$ by $90 \mathrm{ft}$ ) with a table and two chairs in it. The arrangement of the furniture was kept the same throughout the study. The odours were lemon and lavender essential oils, used as in Experiment 1 .

The list of 20 words was derived from Battig and Montague's (1969) categorical norms. The words were divided into five categories. For each category, two of the four words were of low frequencies and the other two were of higher frequencies.

The Tower of Hanoi problem was given first in concrete and then in abstract versions. Both problems were printed on paper and visual aids were provided. In the abstract version, three rings (labelled Small, Medium, Large) are placed in order of size with the largest on the bottom, on the first three pegs (labelled Peg A, Peg B, Peg C). The object of the problem is to transfer all the rings from Peg A to Peg C. There were two constraints: only one ring could be moved at one time and no larger ring could be placed over a smaller one on any peg. The concrete 'Christmas Tree' version was isomorphic: subjects were told that three trees must be decorated (labelled Tree A, Tree B, Tree C). On Tree A, there are three types of decorations put in layers from the inside to the edge of the tree. The order must be that lights are placed in the inner layer, tinsel in the middle layer and ornaments in the outer layer. The subject must move the decorations from Tree A to Tree $\mathrm{C}$, but only move the decorations one layer at a time and without placing an inner-layer decoration on top of an outer one. The maze was reproduced on a sheet of A4 paper with a designated start-point indicated by the letter $\mathrm{S}$. There was a single exit.

\section{Design and procedure}

There were two independent variables: the type of odour at study (session 1) and the type of odour at test (session 2). Each independent variable had two levels. Subjects were exposed to either lemon or lavender during the study session, then to either lemon or lavender odour during the test session. This crossed design generated four experimental conditions: lemon-lemon, lavender-lavender, lemon-lavender, lavender-lemon. The design was a between-group design, so each participant took part in only one of the experimental conditions, but all subjects were required to complete three tasks. There were three dependent variables: the number of words recalled from the word list; the difference in time taken to solve each of the two problems; and the difference in time taken to complete the maze at both sessions. 
Each subject attended both sessions on two consecutive days at the same time, to control for time-of-day effects. They were tested individually using a study-test paradigm with a 24-hour interval. Sessions were carried out throughout the day, with the four conditions counterbalanced. Participants were advised that the purpose of the experiment was to assess the effect of odour on cognitive skills and given written instructions throughout. Each was required to complete three tasks per session. For standardization, written instructions were used throughout the experiment.

In the study session, each subject was required to memorize a word-list, which consisted of 20 words (five categories with four words in each). They were then asked to write down as many words as they could remember (in any order) on a blank sheet of paper. This was done to give a sense of closure in order to prevent the participants from rehearsing the word-list again. However, this data was not used for any analysis because there was an inevitable ceiling effect. For the problem-solving task, subjects were allowed to ask questions about the problem but not its solution. When everything was clarified, the experimenter handed over the visual aids, first the pegs or trees, then the rings or ornaments, all in a fixed predetermined sequence stacked from top to bottom (e.g. Tree A, Tree B, Tree C, Lights, Tinsels, Ornaments). Performance was timed from receipt of the visual aids to completion. Third, a maze sheet was given with the instruction to trace with a pen from the designated start-point inside the maze at point $\mathrm{S}$ to the single exit. Subjects were told that they were allowed to start over and to retrace their paths, as necessary. The time recorded began when they placed the pen at point $\mathrm{S}$ and stopped when they reached the exit. They were then told to expect three different problems in the next session.

In the test session, 24 hours later, the participants were given a sheet of paper with the instructions to write down as many words as they could remember from the wordlist presented in the study session. There was no time limit but they were told to stop when they felt that they were guessing. Second, they were presented with the Tower of Hanoi abstract problem and the same procedure was followed as in the learning session. The time taken to complete was recorded. Third, they were given a second session on the same maze task. The instructions told them that the maze was identical to the one in the previous sessions. The time taken to reach the exit was recorded.

\section{Results}

Recall

Table 2(A) shows that the mean number of words recalled in reinstated conditions (i.e. where subjects had either lavender or lemon for both sessions) was higher than when the words were recalled in the incongruous condition (i.e. where subjects had either lemon at learning and lavender at test, or vice versa). An ANOVA showed no significant main effect on recall performance as a result of having lemon or lavender at either learning or test $(F \mathrm{~s}<2)$. However, there was a significant interaction between odour at study and odour at test, which indicated that there was a facilitative effect of context reinstatement $(F(1,44)=5.02, p<0.03)$.

\section{Problem solving}

For the problem-solving task, a savings score was calculated by dividing the difference in the time taken (in seconds) to complete the problem in each session by the time 
Table 2.

\begin{tabular}{|c|c|c|c|}
\hline \multirow{2}{*}{\multicolumn{2}{|c|}{ Test condition }} & \multicolumn{2}{|c|}{ Study condition } \\
\hline & & Lemon & Lavender \\
\hline \multicolumn{4}{|c|}{$\begin{array}{l}\text { (A) Mean }( \pm \text { SD) words recalled in Experiment } 2 \\
\text { (all } N \mathrm{~s}=12)\end{array}$} \\
\hline & Lemon & $17.0( \pm 2.34)$ & $15.6( \pm 2.75)$ \\
\hline & Lavender & $15.4( \pm 2.91)$ & $17.4( \pm 2.54)$ \\
\hline \multirow{3}{*}{ (B) } & $\begin{array}{l}\text { Mean }(\%, \pm \mathrm{SD}) \text { problem-solving savings scores } \\
\text { in Experiment } 2(\text { all } N \mathrm{~s}=12)\end{array}$ & & \\
\hline & Lemon & $42.75( \pm 22.74)$ & $40.42( \pm 22.99)$ \\
\hline & Lavender & $53.58( \pm 17.37)$ & $64.67( \pm 14.11)$ \\
\hline \multirow[t]{3}{*}{ (C) } & $\begin{array}{l}\text { Mean maze completion savings scores }(\%, \pm \mathrm{SD}) \\
\text { in Experiment } 2(\text { all } N \mathrm{~s}=12)\end{array}$ & & \\
\hline & Lemon & $42.17( \pm 27.17)$ & $31.33( \pm 19.76)$ \\
\hline & Lavender & $37.83( \pm 20.38)$ & $45.75( \pm 13.86)$ \\
\hline
\end{tabular}

taken in the first session calculated for each subject. The time taken to solve the second problem was always faster.

From Table 2(B), the group means suggest that savings were greatest when lavender odour was present at test, irrespective of whether lemon or lavender was present at learning. As the data had heterogeneity of variance a square-root transform was applied, following Tukey's ladder of transformation. The ANOVA confirmed that there was a significant main effect of lavender at test $(F(1,44)=8.56, p<0.01)$. There was no significant main effect for lemon odour and the interaction was not significant, indicating that there was no facilitative effect of context reinstatement.

\section{Maze learning}

For the Porteus maze test, a savings score was calculated by dividing the difference in the time taken (in seconds) to complete the maze in each session by the time taken in the first session. As the data had heterogeneity of variance a square-root transform was applied. An ANOVA showed no significant main effect in savings score as a result of having lemon or lavender at either learning or test $(F s<1)$. However, there was a significant interaction between odour at study and odour at test, consistent with a facilitative effect of context reinstatement $(F(1,44)=2.98, p<0.05)$.

\section{Discussion}

The finding of a facilitation of free recall performance when the same odour was present at both study and test in the explicit memory test is consistent with other literature on context-dependent memory. A number of other studies have shown some effect of odour (e.g. Eich, 1978; Schab, 1990; Smith et al., 1992; Parker and Gellatly, 1997) but, as might be expected, this is not reproducible under all conditions of testing (e.g. Ludvigson and Rottman, 1989), and many factors could be responsible for this 
variation in the effectiveness of ambient odour as a retrieval cue. While the maze learning task showed a similar facilitation, there was no context-dependent effect on problem solving: the results do not support the position that positive transfer is greater in all odour-congruent conditions. This dissociation may reflect some psychological dissociation between the processes necessary to complete the tasks, as might be expected given the known neuropsychological dissociations. The positive effect on spatial memory is consistent with other evidence that spatial memory can be improved by contextual reminders (Chun and Phelps, 1999), including odour (Nigrosh et al., 1995). It may therefore be the case that spatial memory in both its explicit and implicit forms may benefit from the reinstatement of a variety of types of contextual cues.

While we did not see any context-dependent retrieval on the problem-solving task, there was a significant facilitatory main effect of having lavender odour present during the test session, regardless of the odour that had been present at the study session. There was no effect of having lemon odour present at either study or test session. The beneficial effect of the lavender could have been due to reduced test anxiety or some non-specific 'activational' effect of the kind discussed by Schab (1990). Whatever the explanation, the different effect on problem solving between the lavender and lemon conditions suggests that the effect was due to some unique property of the lavender oil, rather than the simple (non-specific) presence of an odour. This beneficial effect of lavender in this task contrasts with the finding of Ludvigson and Rottman (1989) who found that lavender adversely affected cognitive reasoning. Until experiments which use the whole range of tasks under similar conditions are run, however, it is difficult to reconcile these differences in results.

\section{GENERAL DISCUSSION}

In Experiment 1, the reintroduction of the same ambient odour (lemon or lavender) improved performance in both free recall and recognition of a word-list, after a fourweek interval. A further comparison with an additional group showed that memory was not improved by the presence of a different odour. Experiment 2 used the same two odour cues (lemon and lavender) and replicated context-dependent retrieval on a free recall test. A context-dependent improvement in efficiency of relearning a spatial maze task was also seen, but there was no such effect on a test of problem solving.

\section{General effects on learning and memory}

In both experiments, the cross-over design enabled us to separate out (for the present purposes) non-specific effects of odour on learning and memory. In Experiment 1, we saw an effect of odour in the study phase for memory measured by the test of recognition and in Experiment 2, lavender odour facilitated performance of the problem-solving task. These effects should be seen as separate from the effects of odour on context-dependent retrieval. They could result if, for example, the presence of an ambient odour adds novelty to a learning situation and so motivates subjects to pay more attention to the task, or alternatively, if the odour relaxes subjects and reduces test anxiety (cf. Schab, 1990). In the case of lavender, this odour is often described as soothing. For problem solving, the facilitation in performance we found 
in the group who experienced lavender odour at test also indicates that the lack of effect of context reinstatement we found was not due to a ceiling effect.

\section{What are the mechanisms of context-dependent retrieval?}

Apparent context-dependent effects could arise because the group with a different odour at recall was distracted by the novelty of the odour. We suggest this was unlikely given that the lemon and lavender odours used in the study are widely available scents used, for example, in domestic cleaning products. We saw contextdependent retrieval in recall, recognition and spatial learning tasks, but not on problem solving. One explanation for this dissociation could be that the latter task was less sensitive. If so, the demonstration of context-dependent retrieval mediated by odour could require the exclusion of other cues, for example those provided by the materials used in the Tower of Hanoi problem. For example, in Locke's anecdote, the trunk may have functioned as a single effective retrieval cue in the performance chamber when other contextual cues from the dancer's learning room were not available. Alternatively, it is possible that more exposures are necessary to strengthen the association between odour and problem solving, just as Locke's dancer had practised for years with the trunk present so that it became incorporated into his dance routines.

What other factors might be important in achieving a significant difference between reinstated and changed context groups? One important factor, expressed by the outshining hypothesis, is the strength of the cues available to the subject at test. If strong cues are available, for example the words themselves in a recognition test with a short study-test interval, it is unlikely that the more subtle effects of EC reinstatement will be observed. In Experiment 1, the long study-test interval ensured that recognition was at a point where such strong cues were likely to be diminished, resulting in facilitation of context-dependent recognition performance. This has similarities with the experience of incidents of context-cued recognition which occur in everyday life. Although the second exposure to the problem-solving and spatial tasks would result in a recognition memory component, the shorter interval here may have been part of the reason, again in terms of the outshining hypothesis, why we found no effect of context reinstatement in the problem-solving task. It may be the case that similarities between the two versions of this task led to a substantial recognition component and consequent outshining, given the short study-test interval. Similarly, the amount of processing at study is important - intentional learning consistently results in very high recall performance and therefore outshining. We should also consider sample size. Although the number of subjects was enough to capture the effects of context on explicit memory, perhaps a larger sample would be needed to detect any real effects on procedural memory in the problem-solving task if the effect size were smaller. This remains an interesting question for future research.

\section{Practical applications}

It follows that at least for tests of explicit memory, olfactory cues could be used systematically to improve memory. This leads to the idea that odour can be used as a moveable contextual cue, which can be more easily transported than a physical context cue. Parker and Gellatly (1997) investigated the role of odour and music as 
portable cues and found that at least some of the benefits of both context cues are transferable, even when the test environment is physically different. Another possibility is that subjects might even benefit from mentally reinstating the study context at test (see Smith, 1988).

Effective reinstatement of context by any systematic method can be a practical aid to a range of individuals and situations. For example, it has formally been applied to witness interrogation procedures by incorporating it into the Geiselmann's Cognitive Interview procedure (Fisher and Geiselman, 1988) that uses a number of cognitive strategies designed to improve recall accuracy. In particular, odour could be a useful cue as odour-evoked memories have been found to be more vivid (Gibbons, 1986) and emotionally potent (Herz and Cupchik, 1995). Such retrieval cues are also likely to be of benefit in clinical settings, particularly in the management of impaired memory in amnesic patients and the elderly (cf. Winocur et al., 1987). Olfactory information might be of particular benefit in memory retrieval given that odour cues are associated with emotional autobiographical memory and, compared to other cues, they may become stronger with time (Goldman and Seamon, 1992; Parker, 1994; Rubin et al., 1984).

\section{Conclusions and implications}

The results of this series of experiments have shown that memory is enhanced by the addition of distinctive odour cues to the environment. The proposal that environments will be more distinctive when they differ from one another on as many sensory dimensions as possible is hard to refute. Only further research will tell us about the effectiveness of odour as a cue under a range of conditions. Implicit memory in all its forms, in particular, has been surprisingly under-investigated for context-dependent effects. However, as a dissociation can be found between a facilitation of conceptual implicit memory, but no facilitation of perceptual implicit memory with EC reinstatement (Parker et al., 1999), there may be a variety of effects yet to be discovered in this area. Moreover, in naturalistic settings, we are exposed to configurations of cues that change as we move through space. Thus experimental manipulation of cues in isolation will always underestimate the size of context-dependent effects associated with interactions between cues. Experimentally, one could increase the number or complexity of the ambient cues. For example, everyday experience of memories evoked by music that has not been heard for a long time suggests that music may also work in this Proustian fashion. Another possibility which should be further explored is alteration of the study-test interval; in applied settings, this could obviously vary greatly. Odour in particular may become more effective as a cue with the passage of time and the consequent weakening of other types of cue.

\section{REFERENCES}

Aggleton JP, Waskett L. 1999. The ability of odours to serve as state-dependent cues for realworld memories: Can Viking smells aid the recall of Viking experiences? British Journal of Psychology 90: 1-7.

Battig WF, Montague WE. 1969. Category norms for verbal items in 56 categories: a replication and extension of the Connecticut category norms. Journal of Experimental Psychology (Monograph) 80: 1-46. 
Chun MM, Phelps EA. 1999. Memory deficits for implicit contextual information in amnesic subjects with hippocampal damage. Nature Neuroscience 2: 844-847.

Eich JE. 1978. Fragrances as cues for remembering words. Journal of Verbal Learning and Verbal Behavior 17: 103-111.

Eich JE. 1980. The cue-dependent nature of state-dependent retrieval. Memory and Cognition 8: $157-173$.

Engen T, Ross BM. 1973. Long-term memory of odors with and without verbal descriptions. Journal of Experimental Psychology 100: 221-227.

Fernandez A, Glenberg AM. 1985. Changing environmental context does not reliably affect memory. Memory and Cognition 13: 333-345.

Fisher RP, Geiselman RE. 1988. Enhancing eyewitness memory with the cognitive interview. In Practical Aspects of Memory: Current Research and Issues, Vol. 1: Memory in Everyday Life, Gruneberg MM, Morris PE, Sykes RN (eds.). Wiley: Chichester; 34-39.

Gibbons B. 1986. The intimate sense of smell. National Geographic September; 324-360.

Godden DR, Baddeley AD. 1975. Context-dependent memory in two natural environments: on land and underwater. British Journal of Psychology 66: 325-331.

Goldman WP, Seamon JG. 1992. Very long-term memory for odors: retention of odor-name associations. American Journal of Psychology 105: 549-563.

Graf P, Williams D. 1987. Completion norms for forty three letter word stems. Behavior Research Methods, Instruments and Computers 19: 422-445.

Hertz RS, Cupchik GC. 1995. The emotional distinctiveness of odor-evoked memories. Chemical Senses 20: 517-528.

Kucera H, Francis WN. 1967. Computational Analysis of Present-day American English. Brown University Press: Providence, RI.

Laird DA. 1935. What can you do with your nose? Scientific Monthly 41: 126-130.

Lawless HT, Cain WS. 1975. Recognition memory for odors. Chemical Senses and Flavor 1: $331-337$.

Locke J. 1690. An Essay Concerning Human Understanding (Everyman's Library Edition, 1961). Dent: London.

Ludvigson HW, Rottman TR. 1989. Effects of ambient odors of lavender and cloves on cognition, memory, affect and mood. Chemical Senses 14: 525-536.

Lyman BJ, McDaniel MA. 1986. Effects of encoding strategy on long-term memory for odors. Quarterly Journal of Experimental Psychology 38A: 753-765.

Lyman BJ, McDaniel MA. 1990. Memory for odors and odor names: Modalities of elaboration and imagery. Journal of Experimental Psychology, Learning, Memory and Cognition 16: 656664.

Mayes AR. 1988. Human Organic Memory Disorders. Cambridge University Press: Cambridge.

Mayes AR, Meudell PR, Som S. 1981. Further similarities between amnesia and normal attenuated memory: Effects with paired-associate learning and context-shifts. Neuropsychologia 19: 655-664.

Nigrosh BJ, Slotnick BM, Nevin JA. 1995. Olfactory discrimination, reversal learning and stimulus control in rats. Journal of Comparative Psychology 89: 285-294.

Parker A. 1994. Aspects of the Environment: The Effects of Extrinsic Context Cues on Implicit and Explicit Memory. PhD Thesis, Keele University.

Parker A, Gellatly ARH. 1997. Moveable cues: A practical method for reducing contextdependent forgetting. Applied Cognitive Psychology 11: 163-173.

Parker A, Gellatly ARH, Waterman M. 1999. The effect of environmental context manipulation on memory: dissociation between perceptual and conceptual implicit tests. European Journal of Cognitive Psychology 11: 555-570.

Porteus SD. 1965. Porteus Maze Test: Fifty Years of Application. Pacific Books: Palo Alto, CA.

Rubin DC, Groth E, Goldsmith DJ. 1984. Olfactory cuing of autobiographical memory. American Journal of Psychology 97: 493-507.

Schab FR. 1990. Odors and the remembrance of things past. Journal of Experimental Psychology: Learning, Memory and Cognition 16: 648-655.

Smith DG, Standing L, de Man A. 1992. Verbal memory elicited by ambient odor. Perceptual and Motor Skills 74: 339-343. 
Smith SM. 1986. Environmental context dependent recognition memory using a short-term memory task for input. Memory and Cognition 14: 347-354.

Smith SM. 1988. In Memory in Context: Context in Memory, Davis GM, Thompson DM (eds.). Wiley: Chichester; 13-34.

Smith SM, Glenberg A, Bjork RA. 1978. Environmental context and human memory. Memory and Cognition 6: 342-353.

Squire LR. 1992. Declarative and nondeclarative memory: multiple brain systems supporting learning and memory. Journal of Cognitive Neuroscience 4: 232-243.

Winocur G, Moscovitch M, Witherspoon D. 1987. Contextual cueing and memory performance in brain-damaged amnesic and old people. Brain and Cognition 6: 129-141. 